\title{
The Development of Metal Oxide Chemical Sensing Nanostructures
}

\author{
G. W. Hunter ${ }^{\mathrm{a}}$, R. L. Vander Wal ${ }^{\mathrm{b}}$, J. C. Xu ${ }^{\mathrm{a}}$, L. J. Evans ${ }^{\mathrm{a}}$, G. M. Berger ${ }^{\mathrm{b}}$ and M.J. Kulis ${ }^{\mathrm{b}}$ \\ a) NASA Glenn Research Center Cleveland, $\mathrm{OH} 44135$ \\ b) USRA at NASA Glenn Research Center, Cleveland, OH 44135
}

This paper discusses sensor development based on metal oxide nanostructures and microsystems technology. While nanostructures such as nanowires show significant potential as enabling materials for chemical sensors, a number of significant technical challenges remain. This paper discusses development to address each of these technical barriers: 1) Improved contact and integration of the nanostructured materials with microsystems in a sensor structure; 2) Control of nanostructure crystallinity to allow control of the detection mechanism; and 3) Widening the range of gases that can be detected by fabricating multiple nanostructured materials. A sensor structure composed of three nanostructured oxides aligned on a single microsensor has been fabricated and tested. Results of this testing are discussed and future development approaches are suggested. It is concluded that while this work lays the foundation for further development, these are the beginning steps towards realization of repeatable, controlled sensor systems using oxide based nanostructures.

\section{Introduction}

Application of metal-oxide semiconductors (such as semiconducting tin oxide) in chemical sensing applications has been on-going for a number of years. These applications include environmental monitoring, fire detection, and emissions monitoring. The fundamental sensing mechanism of these metal oxide based gas sensors relies upon the change in electrical conductivity due to the interaction between the gases in the environment and adsorbates (e.g. oxygen) in the grain boundaries. The use of nanocrystalline material, i.e. materials with grain size on order of nanometers $(\mathrm{nm})$, results in improved sensor sensitivity, stability, and response time. This technology has reached a high level of maturity including the capability to reproducibly fabricate the nanocrystalline materials and integrate them into operational sensor structures [1-2].

However, the ability to fabricate operational sensors from nanostructures is significantly less mature. Nanostructures, e.g. nanowires, nanotubes, and nanobelts, have fundamentally different structural properties than nanocrystalline grains. The fabrication procedures, properties, and possible advantages of nanostructured oxide sensors are just beginning to be explored. This paper discusses sensor development that focuses on moving from nanocrystalline materials toward nanostructures as the active sensing material. Based on the dramatic improvements in sensor performance demonstrated by moving from macrograined to nanograined material, investigations are under way to determine if similar gains in sensor performance can be achieved by use of these nanostructures [3]. 
Development of these nanostructured sensors by this group is being approached by implementing MEMS (Micro-Electro-Mechanical Systems) or Microsystems technology [1-4] in combination with the control of the fabrication of the nanostructured materials. The MEMS structures themselves are modified in order to assist in the alignment of the nanostructures, vary sensor response, produce sensor arrays, and fabricate operational systems. The basic ability to control the orientation and alignment of nanostructures on microstructures is still in its early stages although various groups have made some progress [5-8].

Our activities focus on efforts to address three technical barriers that have been identified as fundamental to the application of nanostructures into sensor systems [3]. The overall viewpoint is that although nanotechnology has significant potential, the basic tools to make reproducible sensors that can employ different sensing mechanisms (as is done in microtechnology) are not yet available. The three areas of emphasis are: 1) Improving the contact and integration of the nanostructured materials with microsystems in a sensor structure; 2) Controlling nanostructure crystallinity to allow control of the detection mechanism; and 3) Widening the range of gases that can be detected by using different nanostructured materials.

This paper discusses an example sensor structure and provides an overview of the present status of this work. This example discusses the fabrication and testing of a single sensor structure that includes three different nanostructured oxide materials aligned on a microplatform. Conclusions from this example and future directions are described. This work demonstrates useful tools which with further development can lead to the ability to form repeatable, operational sensors. However, process control and optimization of the sensors for a given set of gases and operational environments is not yet mature. This includes a lack of a clear understanding of sensing mechanisms and correlation of sensing properties to processing. Significant further work is necessary towards the realization of repeatable, controlled sensor systems composed of oxide based nanostructures.

\section{Experimental}

The metal oxide semiconductors discussed in this work are synthesized by a thermal evaporation-condensation (TEC) approach [4]. A metal oxide nanostructure was fabricated beginning with evaporating the corresponding metal within the flow of an inert gas plus a minute amount of oxygen. Typical processing conditions include a temperature of $\sim 1000^{\circ} \mathrm{C}$ and a flow of an inert gas such as argon or nitrogen. Alternative approaches have included carbothermal reduction of the oxide mixed with powdered graphite in either volumetric or molar ratios of 1:1. Subsequently oxide materials are collected from the deposition substrate or boat and dispersed within a liquid for subsequent deposition upon the sensor interdigitated pattern.

For example, to produce zinc oxide $(\mathrm{ZnO})$, an alumina boat holds the zinc powder within a quartz tube placed horizontally within a tube furnace maintained at $500^{\circ} \mathrm{C}$ or above. In the absence of catalysts, growth occurs via a vapor-solid (VS) mechanism although an oxide assisted mechanism may also contribute. Nanowires, nanoblades, or tetrapods may be formed depending upon the details of the furnace temperature, gas flow 
rate, and temperature of the collection zone [9]. Zinc oxide nanostructures were collected downstream from plates positioned at lower temperature regions. Tin dioxide $\left(\mathrm{SnO}_{2}\right)$ nanostructures were similarly formed from $\mathrm{SnO}$ powder held within an alumina boat but evaporated species form nanowires within the same boat, along the boat edges, and on the surface of the source material. Indium oxide $\left(\operatorname{In}_{2} \mathrm{O}_{3}\right)$ nanostructured materials were fabricated in a similar fashion.

The microsensor platforms were fabricated on polished alumina substrates using standard photolithography techniques. Commercially available alumina substrates (625 $\mu \mathrm{m}$ in thickness) [10] were patterned with photoresist to create an interdigitated finger mask. Titanium $(50 \AA)$ and platinum $(2500 \AA)$ were sequentially deposited on the substrate by sputtering. After lift-off, the substrates with electrodes were diced into individual devices.

Dielectrophoresis was used to align the nanowires so as to bridge the electrodes. Dimethylformamide (DMF) was found to form a relatively stable suspension of metal oxide nanostructures and was also compatible with subsequent dielectrophoresis. Using a pipette, a droplet of the suspension was placed upon an interdigitated grid. In this process, an $\mathrm{AC}$ voltage was applied across the electrode grid using a function generator. For nanowires less than 10 microns long, $10 \mathrm{~V} \mathrm{AC}$ at a frequency of $5 \mathrm{MHz}$ was applied. For nanowires greater than 10 microns, a lower frequency appears to improve alignment. For example, lowering the frequency from $5 \mathrm{MHz}$ to $500 \mathrm{KHz}$ appeared to improve the alignment of $\mathrm{SnO}_{2}$ nanowires that had a length greater than 20 microns long.

After alignment by dielectrophoresis, the solvent was allowed to evaporate while the voltage was applied to the grid. The resistance across the grid was measured after the solvent completely evaporates. Typically a measurable resistance (less than $10 \mathrm{M} \Omega$ ) was found after 4 drop/evaporation cycles are completed. After each deposition step, the nanowire placement on the interdigitated grid was observed using an optical microscope to verify deposition uniformity of nanowires. Structural characterization of the nanowires was performed with transmission electron microscopy (TEM) and morphology with scanning electron microscopy (SEM). Energy dispersive spectroscopy by X-ray (EDS) confirmed the elemental compositions of the nanostructured forms as being stoichiometric $\mathrm{SnO}_{2}, \mathrm{ZnO}$, or $\mathrm{In}_{2} \mathrm{O}_{3}$.

The sensors were tested in a chamber with the sensor temperature controlled on a heater stage and electrical contact was made with probes. Gases were introduced into the chamber at a total flow rate of 4000 standard cubic centimeters per minute $(\mathrm{sccm})$. Different concentrations of gases were introduced at temperatures up to $400^{\circ} \mathrm{C}$. For 5 minutes at each step, the sensor was first exposed to air, nitrogen, $0.5 \%$ hydrogen in nitrogen, nitrogen, air, and then $0.5 \%$ ethylene in nitrogen followed by air. The response of the sensor was measured by current output at a constant voltage of $1 \mathrm{~V}$. Current-voltage (I-V) curves were taken periodically to examine sensor response over a range of voltages.

\section{$\underline{\text { Overview }}$}

\section{Sensor Development}

As part of efforts to improve micro-nano contacts and integration, control of oxide 
nanostructure crystallinity, and expand the range of available nanostructured materials, previous work has demonstrated the capability to align an oxide nanostructure on a microplatform using dielectrophoresis [4]. The oxide nanostructure discussed was zinc oxide $(\mathrm{ZnO})$ aligned across parallel, interdigitated fingers. This work showed the fundamental capability to align a nanostructure on a microplatform. The fabrication of multiple nanostructures such as $\mathrm{ZnO}$, tin oxide $\left(\mathrm{SnO}_{2}\right)$, indium oxide $\left(\mathrm{In}_{2} \mathrm{O}_{3}\right)$, and iron oxide $\left(\mathrm{Fe}_{2} \mathrm{O}_{3}\right)$ was also demonstrated with the corresponding capability to fabricate both single and polycrystalline materials [4].

This section discusses an example that combines all three aspects of this work and extends the capabilities discussed in reference 4 . As part of a longer-term vision to better control the fabrication of sensor structures using nanostructured oxides, the fabrication and alignment of 3 different oxide nanostructured materials on the same microplatform is demonstrated. The nanostructures chosen were $\mathrm{SnO}_{2}, \mathrm{ZnO}$, and $\mathrm{In}_{2} \mathrm{O}_{3}$. Each of these three nanostructured materials was chosen for their viability as sensing materials. While each has individually been aligned in a microplatform previously, this current work demonstrated the alignment of all three in a single platform. The ability to align and control multiple materials on a microplatform is a fundamental step towards controlling, understanding, and modeling nanostructured sensors.

\section{Microsensor Fabrication and Material Characterization}

Figure 1 shows the three materials at different locations on the same microsensor platform. The microsensor platform was composed of interdigitated fingers, but not with parallel, linear fingers. Rather, a saw tooth interdigitated sensor pattern was used as shown in Figure 1. The fingers of the pattern are fabricated so that the "points" and recesses of sawtooth fingers align with each other. This was done in order to maximize the effect of the electric field on aligning the nanostructures by dielectrophoresis. Different spacing and nanostructure sizes have been used. Figure 1 shows a saw-tooth pattern fabricated with titanium electrodes having $30 \mu \mathrm{m}$ spacing between the parallel electrodes. The bridging of the various nanostructures across the fingers is visible in the figure.

a)

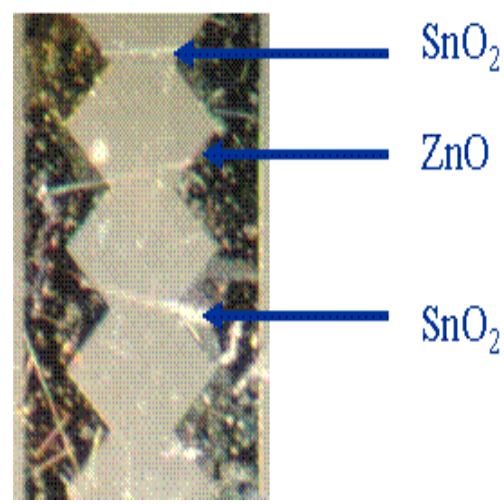

b)

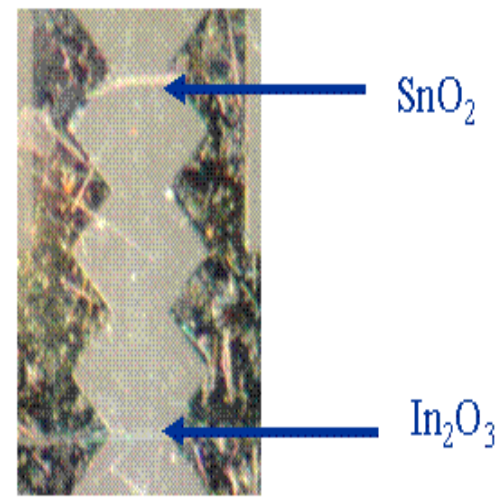

Figure 1. Three nanostructured oxides $\left(\mathrm{SnO}_{2}, \mathrm{ZnO}\right.$, and $\left.\mathrm{In}_{2} \mathrm{O}_{3}\right)$ aligned on the same microplatform. The sawtooth pattern spacing is $30 \mu \mathrm{m}$. These optical pictures are taken at various locations on the microstructure.

The approach used to fabricate the structure in Figure 1 was to first fabricate the 
nanostructures by TEC, and then to incorporate the nanostructures onto the microstructure. The nanostructures were sequentially added onto the microstructure. For example, first $\mathrm{SnO}_{2}$ was aligned independently by dielectrophoresis as described in the Experimental section. After evaporation of the solvent and identification of the position of the aligned nanostructures, the deposition of $\mathrm{ZnO}$ was performed on the same substrate, adjusting the dielectrophoresis parameters appropriately for the material. The fabrication of the structure is completed in the same fashion by evaporation, nanostructure position identification, and the final deposition and alignment of $\operatorname{In}_{2} \mathrm{O}_{3}$.

a)

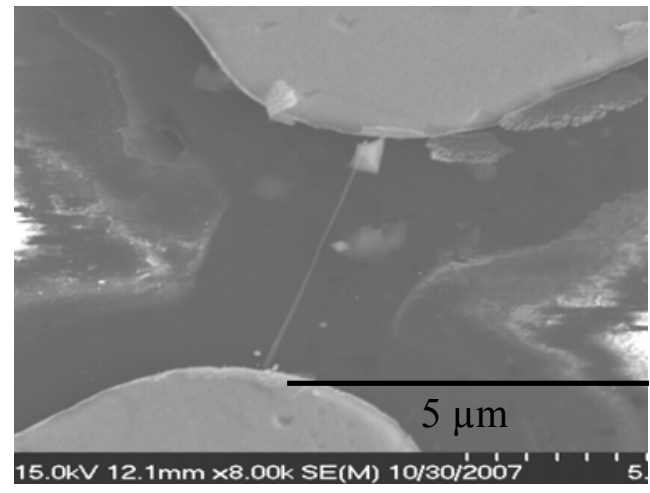

c)

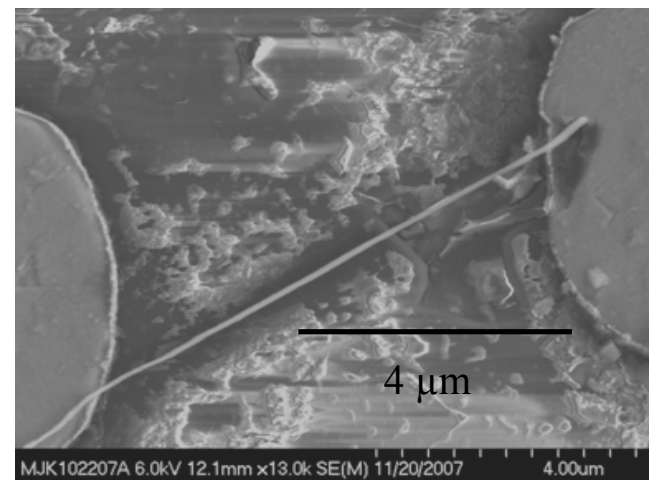

b)

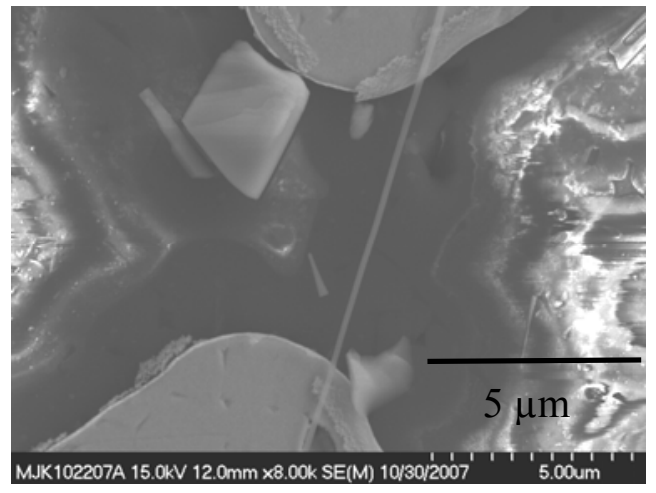

d)

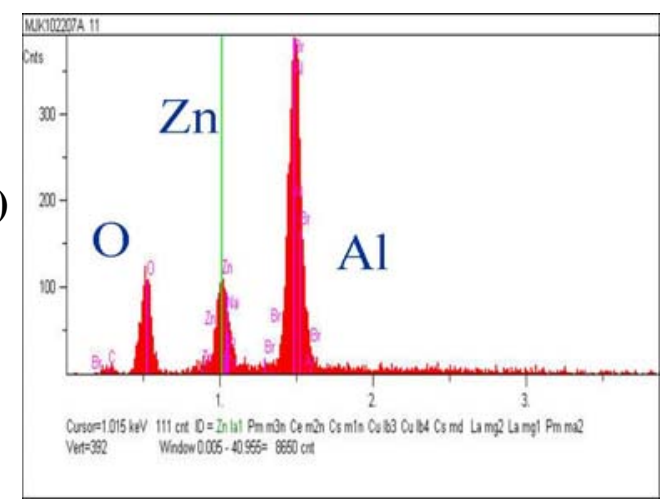

Figure 2. SEM micrographs of three different oxide nanostructures of various sizes on a single $\mathrm{Al}_{2} \mathrm{O}_{3}$ substrate. a) $\mathrm{SnO}_{2}$ nanowire with a diameter of $60 \mathrm{~nm}$. b) $\mathrm{In}_{2} \mathrm{O}_{3}$ nanowire with a diameter of $140 \mathrm{~nm}$. c) $\mathrm{ZnO}$ nanowire with a diameter of $80 \mathrm{~nm}$. d) Corresponding EDS analysis of the $\mathrm{ZnO}$ nanowire in Figure $2 \mathrm{c}$ verifying the elemental configuration of the nanowire and substrate.

The nanostructures shown in Figure 1 are in the higher diameter range of what is considered nanotechnology, with diameters in the range from 300-500 nm. This accounts for the ability to see both the interdigitated finger structure and multiple bridging oxides on the same optical picture as shown in the figure. To demonstrate the applicability of this technique to work on a range of nanostructure diameters, the alignment of nanostructures of multiple sizes has been demonstrated. On a separate microstructure than that of Figure 1, the three nanostructured oxides $\mathrm{SnO}_{2}, \mathrm{ZnO}$, and $\mathrm{In}_{2} \mathrm{O}_{3}$ with a range of smaller diameters were deposited on a sawtooth interdigitated structure. The bridging of these nanostructures across several electrodes is shown in Figure 2 for each material type. Confirmation that $\mathrm{ZnO}$ has indeed been deposited on the microplatforms was accomplished by EDS as shown in Figure $2 \mathrm{~d}$ (corresponding to the $\mathrm{ZnO}$ nanowire in Figure 2c). Corresponding EDS measurements have been taken for the other nanowire 
materials to verify material composition (not shown). The diameters of the oxide nanostructures shown in Figure 2 range from 60-140 nm.

Further, a range of nanostructure diameters have been fabricated and deposited for each oxide material. Examples are shown in Figure 3 corresponding to various $\mathrm{SnO}_{2}$ diameters on this same microplatform. Along with the alignment and bridging across electrodes shown in Figure 2 of a $60 \mathrm{~nm}$ diameter $\mathrm{SnO}_{2}$ nanowire, the alignment of 70 $\mathrm{nm}$ and $125 \mathrm{~nm}$ diameter $\mathrm{SnO}_{2}$ nanowires is shown for elsewhere on the same substrate in Figure $3 \mathrm{a}$ and $3 \mathrm{~b}$. A fundamental point of this work is that control of the dielectrophoresis process, e.g. varying voltage and frequency, yields limited but definable control over the materials aligned and deposited. It should be noted that the materials discussed in this work are single crystal. Verification of this is shown in Figure $3 \mathrm{c}$ for the starting $\mathrm{SnO}_{2}$ material, and similar data has been taken for the $\mathrm{ZnO}$ and $\mathrm{In}_{2} \mathrm{O}_{3}$ nanostructures used in this work. It is suggested that different dielectrophoresis operational parameters may be necessary if polycrystalline materials are used.

a)

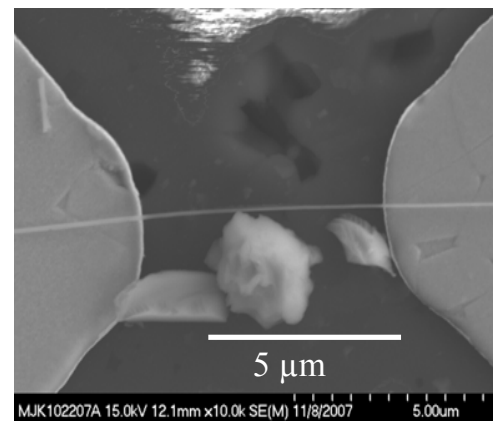

b)

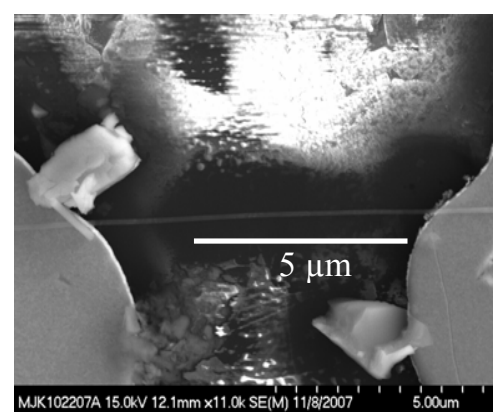

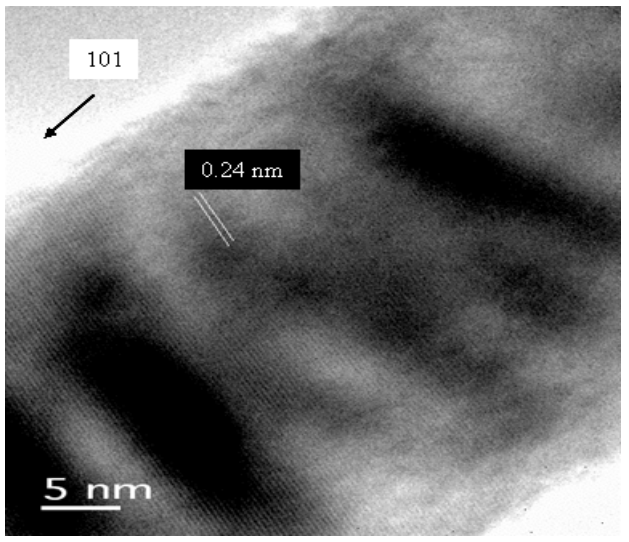

c)

Figure 3. SEM micrographs of $\mathrm{SnO}_{2}$ nanostructures taken elsewhere on the microplatform of Figure 2 showing alignment of $\mathrm{SnO}_{2}$ nanowires of a range of diameters. a) $\mathrm{SnO}_{2}$ nanowire with a diameter of $70 \mathrm{~nm}$. b) $\mathrm{SnO}_{2}$ nanowire with a diameter of $125 \mathrm{~nm}$. c) TEM of the $\mathrm{SnO}_{2}$ starting materials showing the single crystal nature of the TEC fabricated $\mathrm{SnO}_{2}$.

Thus, this work has shown the ability to simultaneously align multiple nanostructured oxide materials on a single microplatform. The nanostructures are single crystal and a range of sizes can be deposited on a single substrate. The pattern of the microplatform, i.e., interdigitated fingers with a sawtooth pattern with an order of microns in spacing, has been significant in allowing these results. Thus, this work shows in a single microsensor platform: 1) Improved control and alignment of the contact between nanostructured materials and electrodes in a microsensor structure. 2) Controlling of nanostructure crystallinity centering on the fabrication and alignment of single crystal 
nanostructures. and 3) Simultaneous alignment of three different nanostructured materials on a single microsensor structure which in a single platform combines the gas sensitivity of three materials.

\section{$\underline{\text { Sensor Testing }}$}

The gas sensing properties of the sensor structure of Figure 1, i.e., the sensor structure with $\mathrm{SnO}_{2}, \mathrm{ZnO}$, and $\mathrm{In}_{2} \mathrm{O}_{3}$ nanostructured oxides of nanowire diameters between 300$500 \mathrm{~nm}$ aligned across sawtooth interdigitated fingers with $30 \mu \mathrm{m}$ spacing, were tested at a range of temperatures and gas concentrations. The temperature was varied between room temperature and $400^{\circ} \mathrm{C}$ with exposure to air, nitrogen, hydrogen, and ethylene as described in the experimental section above.

The data shown in Figure 4 compares the results of this testing at the various temperatures and gas exposures. Table 1 summarizes the sensor response of the nanostructures for the four temperatures. All four temperatures show a response to both hydrogen and ethylene. The baseline response is generally constant, but drift in the baseline is noted with the largest change in baseline evident after exposure to hydrogen at $200^{\circ} \mathrm{C}$. The sensor response to both hydrogen and ethylene is small at room temperature. The sensor response to both gases increases with temperature until $400^{\circ} \mathrm{C}$, at which point a significant drop in response, and a generally less stable signal, is noted. Discontinuities of sensor signal, e.g., near 34 minutes in Figure $4 \mathrm{c}\left(200^{\circ} \mathrm{C}\right)$, typically correspond to times at which I-V curve data was taken. Drift in the signal is seen in pure nitrogen at all temperatures above room temperature, but this drift nearly stabilized within 5 minutes at 300 and $400^{\circ} \mathrm{C}$. The most rapid response to hydrogen and ethylene is evident at 300 and $400^{\circ} \mathrm{C}$.

\begin{tabular}{|c|c|c|c|c|c|c|}
\hline $\begin{array}{c}\text { Temperature } \\
\left({ }^{\circ} \mathrm{C}\right)\end{array}$ & $\begin{array}{c}\text { Max } \\
\text { Starting Air } \\
\text { Baseline } \\
(\mathrm{nA})\end{array}$ & $\begin{array}{c}\text { Max } \mathrm{N}_{2} \\
\text { current } \\
(\mathrm{nA})\end{array}$ & $\begin{array}{c}\text { Max } \mathrm{H}_{2} \\
\text { Current } \\
(\mathrm{nA})\end{array}$ & $\begin{array}{c}\text { Change in } \\
\text { Max H}_{2} \\
\text { current from } \\
\text { Air Baseline } \\
(\mathrm{nA})\end{array}$ & $\begin{array}{c}\text { Max } \\
\text { Ethylene } \\
\text { Current } \\
(\mathrm{nA})\end{array}$ & $\begin{array}{c}\text { Change in Max } \\
\text { Ethylene current } \\
\text { from Air Baseline } \\
(\mathrm{nA})\end{array}$ \\
\hline 25 & 0.58 & 0.39 & 10.00 & 9.42 & 3.40 & 2.82 \\
\hline 100 & 23.94 & 31.42 & 47.53 & 23.59 & 37.48 & 13.54 \\
\hline 200 & 15.89 & 27.90 & 45.56 & 29.67 & 43.82 & 27.93 \\
\hline 300 & 7.42 & 33.56 & 126.08 & 118.66 & 25.72 & 18.3 \\
\hline 400 & 1.97 & 11.75 & 42.60 & 40.63 & 10.45 & 8.48 \\
\hline
\end{tabular}

Table 1. Summary of the results of Figure 4 of the multiple nanostructured oxide microsensor. The maximum (Max) current of the various gases at temperature from $25^{\circ} \mathrm{C}$ to $400^{\circ} \mathrm{C}$ is presented. Sensor response at $300^{\circ} \mathrm{C}$ is shown to have noticeably different behavior than at other temperatures especially in the response to $\mathrm{H}_{2}$.

The preliminary conclusion from the data in Figure 4 and Table 1 is that the optimum sensor operating temperature for this structure is approximately $300^{\circ} \mathrm{C}$. The sensor responses increase from room temperature peaking at $300^{\circ} \mathrm{C}$, with the largest and most rapid sensor response to hydrogen, and the second largest response to ethylene, exhibited at this temperature. This is combined with a generally stable baseline. Further work would be necessary to completely optimize sensor response. It also appears that the sensor degrades as it is heated to $400^{\circ} \mathrm{C}$. In particular, when compared to the $300^{\circ} \mathrm{C}$ re- 
sponses, the sensor response at $400^{\circ} \mathrm{C}$ has a decreased baseline current (increased overall circuit resistance) and a decreased magnitude of sensor response to hydrogen and ethylene.

Possible reasons for this decrease in sensor response above $300^{\circ} \mathrm{C}$ include chemical reactions occurring at $400^{\circ} \mathrm{C}$, especially in the contacts between the nanostructured oxides and the electrodes. That is, examination of Figure 1-3 show that the electrical contact between the nanostructures and electrodes appears to be based on surface contact. Higher temperatures can cause chemical reactions in these contact areas, possibly changing the mechanical and chemical nature of these contacts from that formed as-deposited by dielectrophoresis. Further study of this effect, as well as determination of possible methods to improve device contacts, are on-going.

a)
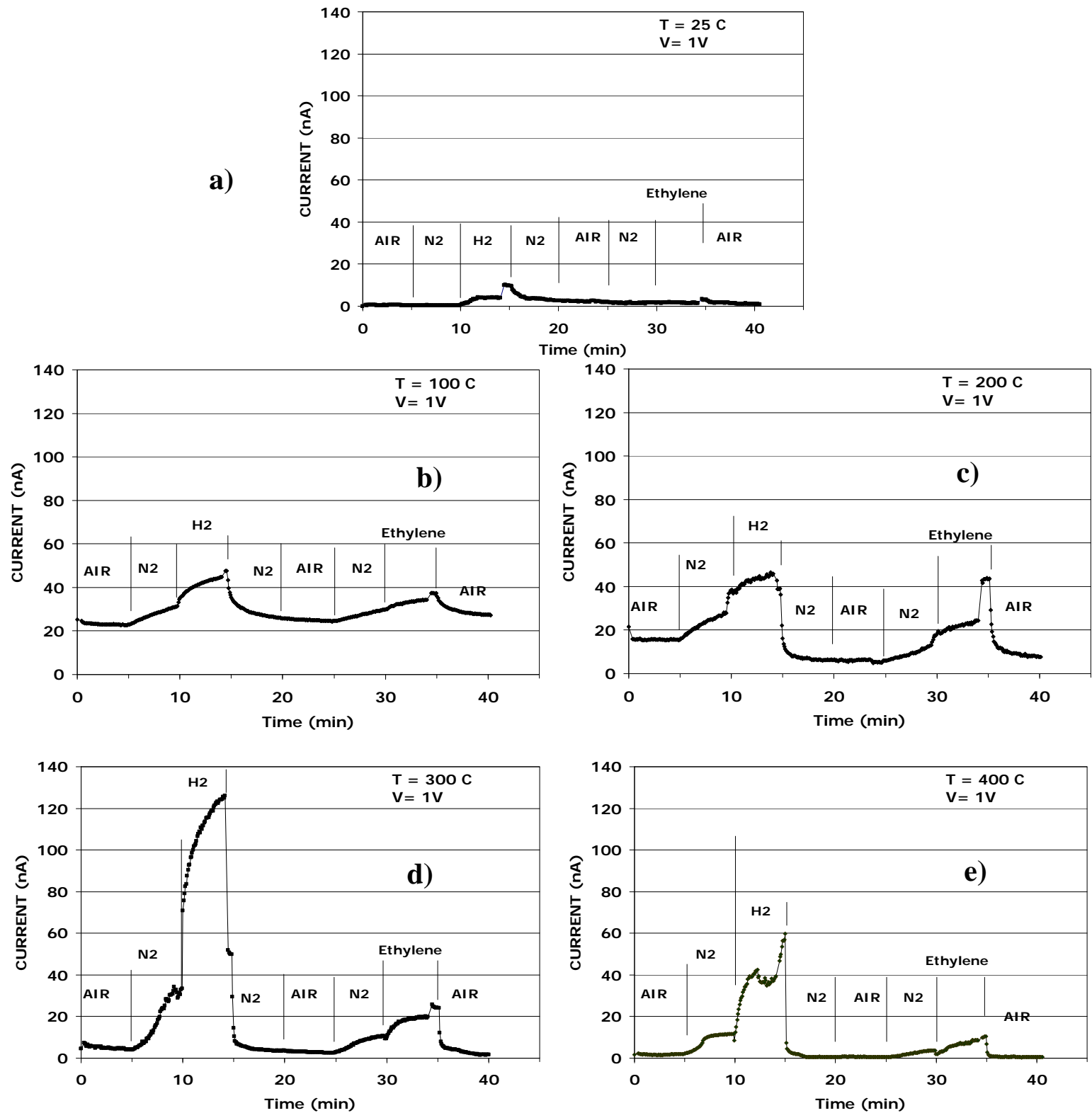

Figure 4. Response vs. time of the microsensor in Figure 1 composed of multiple nanostructured oxides to air, nitrogen $\left(\mathrm{N}_{2}\right), 0.5 \%$ hydrogen $\left(\mathrm{H}_{2}\right)$, and $0.5 \%$ ethylene $\left(\mathrm{C}_{2} \mathrm{H}_{4}\right)$ at a range of temperatures: a) $25^{\circ} \mathrm{C}$; b) $100^{\circ} \mathrm{C}$; c) $200^{\circ} \mathrm{C}$; d) $300^{\circ} \mathrm{C}$; and e) $400^{\circ} \mathrm{C}$. All results are plotted on the same scale for comparison purposes. 
The sensor response at $300^{\circ} \mathrm{C}$ was examined in more detail in Figure 5 where the I-V curves of the sensor are shown for air, $0.5 \%$ hydrogen, and $0.5 \%$ ethylene. Figure $5 \mathrm{a}$ shows the sensor response from $-5 \mathrm{~V}$ to $5 \mathrm{~V}$ while the Figure $5 \mathrm{~b}$ presents the same data but concentrating on the $-1.5 \mathrm{~V}$ to $1.5 \mathrm{~V}$ region. In Figure $5 \mathrm{~b}$, straight lines are fit to the data for illustration purposes. It should be noted that for a resistor, the I-V curve is a straight line, while for an ideal rectifying Schottky diode no current flows in the reverse direction and the current increases exponentially in the positive direction.

The sensor I-V curves differ for each gas. For air, although some asymmetry exists between the reverse and forward current directions, the I-V curve is nearly resistive, i.e., a single nearly straight line encompasses most of the data points for this curve. This is seen by behavior of the air I-V curve in Figure 5a and this point is amplified by the red line in Figure 5b. For hydrogen, the I-V curve is asymmetric and a rectifying effect is observed where the slope of the I-V curve in the positive direction is larger than that in the negative direction as seen in Figure 5a. This point is amplified by the two blue lines of different slope in Figure 5b. In both current directions, there is an observable difference in the magnitude of the current at a given voltage in the hydrogen and air I-V curves.

a)

Figure 5. Current-voltage curves of the microsensor composed of multiple nanostructured oxide sensors in air, $0.5 \% \mathrm{H}_{2}$, and ethylene at $300^{\circ} \mathrm{C}$ for a) $-5 \mathrm{~V}$ to $5 \mathrm{~V}$ and $\mathrm{b}$ ) the same data in the $-1.5 \mathrm{~V}$ to $1.5 \mathrm{~V}$ region with best-fit lines shown.

b)
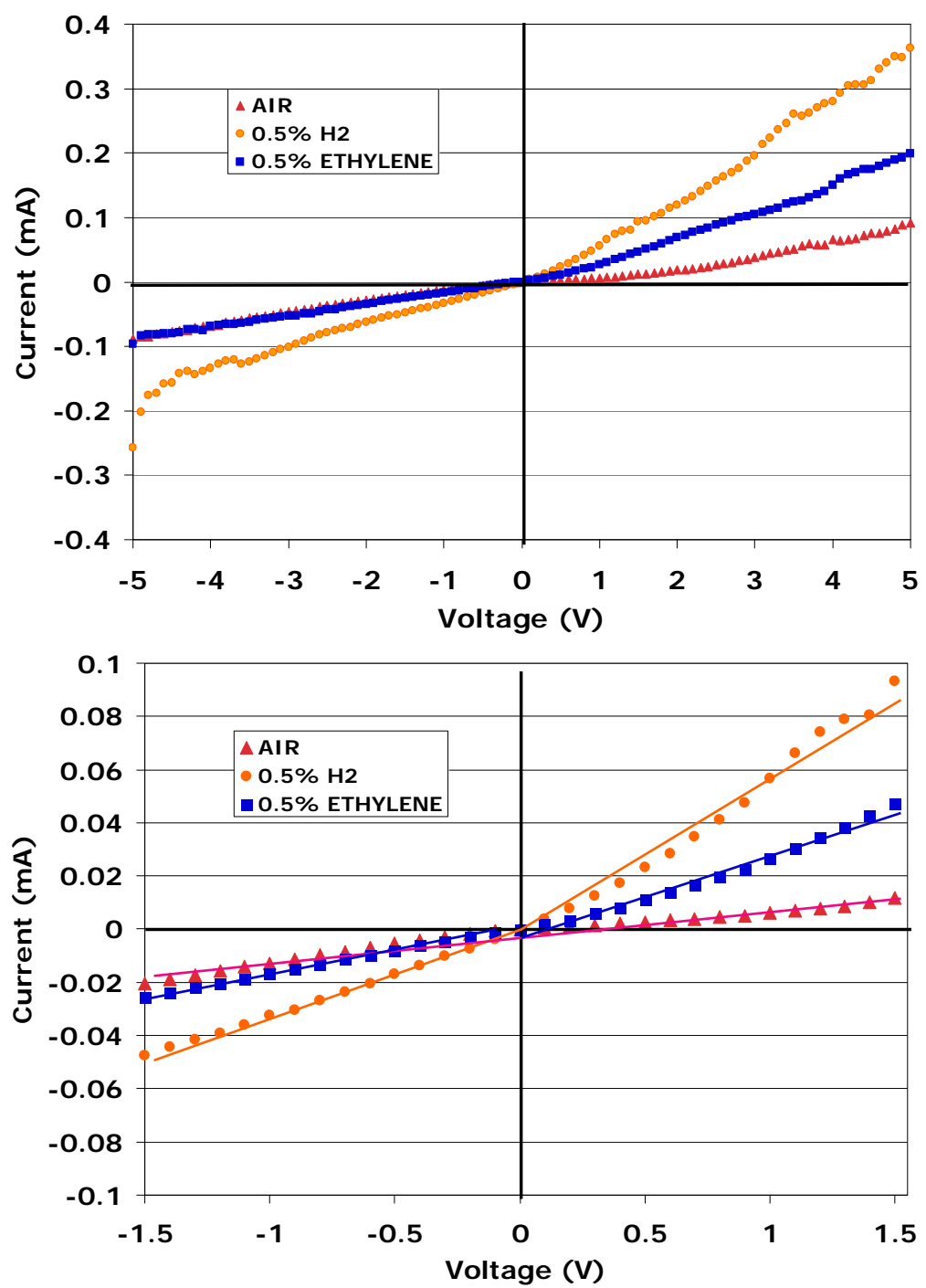

Finally, for ethylene, a rectifying effect is again observed where the slope of the I-V 
curve in the positive direction is larger than that in the negative direction as seen in Figure 5a. This point is amplified by the two orange lines of different slope in Figure 5b. However, in the case of ethylene, while the difference in the ethylene and air curves is observable in the positive current direction, the response to ethylene in the reverse direction is much smaller and the air and ethylene curves nearly overlap. That is, in the reverse current direction, the sensor detects hydrogen, but is nearly insensitive to ethylene. It should be noted that although the behavior of the I-V curves varied with temperature (e.g., I-V curves at $100^{\circ} \mathrm{C}$ exhibited more resistor-like behavior for all gases than I-V curves at $300^{\circ} \mathrm{C}$ ), a common factor at each temperature is that asymmetry is observed between the forward and reverse current flow directions (not shown).

Thus, examination of the I-V curves suggests that the behavior of this sensor structure is temperature dependent, and an asymmetric, rectifying behavior is consistently observed. At this time, the origin of the rectifying behavior is not clear. The nature of the contacts between the nanostructures and the microplatforms may be a possible cause, or the rectifying behavior could be caused by the basic material properties of one, or all, of the nanostructured oxides. Nonetheless, this data does suggest that choice of both temperature and voltage can optimize sensor response, and even affect sensor selectivity.

\section{Summary and Future Directions}

In order to move nanotechnology from a developing technology into operational sensor systems with capabilities and processing uniformity comparable to that available in microtechnology, fundamental technology advances are necessary. Our group has been focusing efforts on addressing the technology challenges of: 1) Improved contact and integration of the nanostructured materials with microsystems in a sensor structure; 2) Control of nanostructure's crystallinity to allow control of the detection mechanism; and 3) Widening the range of gases that can be detected by fabricating multiple nanostructured materials.

This paper discusses an example sensor structure that incorporates all three aspects of this work. Single crystal oxide nanostructures were fabricated and aligned on a microsensor platform using dielectrophoresis. The sensor platform was designed to improve the control of the movement of nanostructures on the microplatform. Three different nanostructured oxide materials were aligned on the same microplatform, and the capability to fabricate and align a range of nanostructure diameters was demonstrated. Testing of the sample suggested the capability to detect hydrogen and ethylene over a wide temperature range, as well as sensitivity to changes in oxygen concentration. Asymmetry in the I-V curves was noted at all temperatures for multiple gases suggesting a rectifying effect possibly due to the nanostructure electrical contacts or the starting material. The optimum operating temperature for the measurement of hydrogen or ethylene for this sensor structure was found to be at $300^{\circ} \mathrm{C}$, with sensor degradation noted upon heating to $400^{\circ} \mathrm{C}$.

Thus, for the first time to our knowledge, a sensor structure composed of three nanostructured oxides aligned on a single microsensor has been fabricated and tested. While further studies are in order to determine the relative contribution of each of the 
oxide nanostructures to the overall sensor response, this work demonstrates a basic capability to fabricate and align multiple oxide nanostructures on sensor microstructures.

This work is intended to be a beginning step towards more complex sensor structures. A number of questions and technical challenges still remain. These include: a) Characterization of the basic properties of the oxide nanostructures individually to understand their basic sensing properties and the cause of the rectifying behavior observed in the sample in this work; b) Improve the fabrication of the microstructures to allow optimization of the uniformity and density of nanostructure alignment, including optimization of the spacing and patterning of the interdigitated fingers; c) Improve the electronic contact between the nanostructures and the electrodes of the interdigitated fingers. These activities are directly related to the sensor structure shown in Figure 1 discussed in this work. Each one is a considerable technical challenge.

For example, the general technical challenge related to improving the electrical contact between the nanostructures and electrodes might be accomplished in a number of ways. One approach would be to deposit metal over electrodes in contact with a nanostructure, i.e., deposit metal over only the electrodes seen in Figure 1 "burying" the electrodes (see for example reference 11).

Another approach is to grow nanostructured oxides directly from the electrodes, thus insuring intimate contact between electrode and nanostructure. Previously we have shown for demonstration purposes the growth of carbon nanotubes from electrodes, where these nanotubes bridge the spacing between the electrodes [4]. This approach has been extended to oxides as is shown in Figure 6. Titanium dioxide $\left(\mathrm{TiO}_{2}\right)$ were grown using controlled oxidation of a titanium grid pattern at $750^{\circ} \mathrm{C}$ with a mixture of $100 \mathrm{sccm}$ acetone in $\mathrm{N}_{2}$ for 4 hours. Figure 6 a shows an overview of the electrode pattern and Figure $6 \mathrm{~b}$ shows the bridging of the nanowires across the electrodes.

Although this growth approach assures strong nanostructure contact on one electrode, controlling the growth of the bridging nanowire to insure strong contact with the other electrode is problematic. Thus, a combination approach of both growth and "burying" of the contact might allow improved electrode and nanostructure electrical contact.

a)

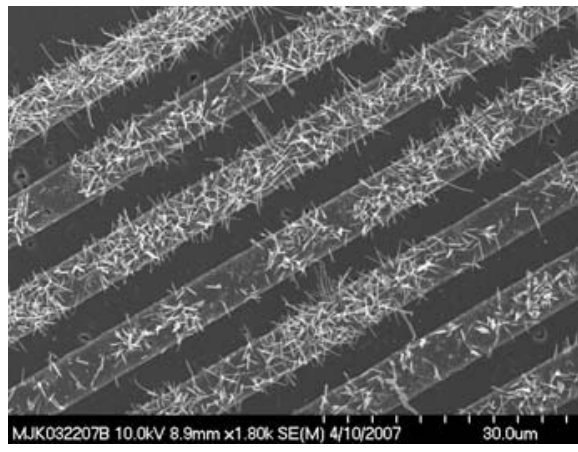

b)

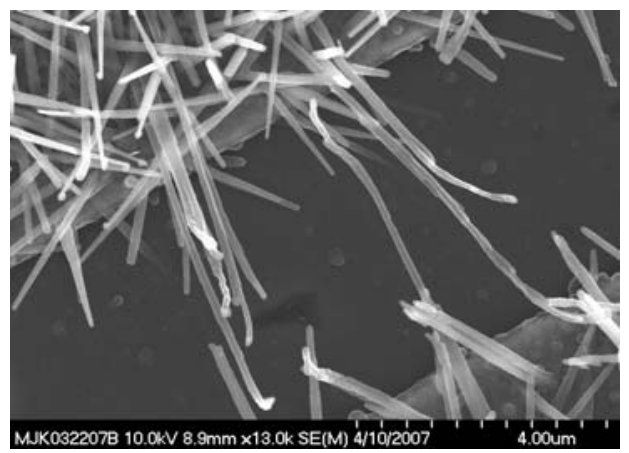

Figure 6. Titanium dioxide nanowires were grown on and across interdigitated fingers: a) A number of interdigitated fingers showing deposition of $\mathrm{TiO}_{2}$ across the sensor structure. b) Bridging of the $\mathrm{TiO}_{2}$ nanowires across the electrodes. 
Overall, in order to address the problems of micro-nano contacts and integration, control of oxide nanostructure crystallinity, and expanded range of nanostructured materials available, the development of a broad range of capabilities will be necessary. This work demonstrates useful tools for further development which are intended to lead to the ability to form repeatable, operational sensors. However, process control and optimization of the sensors for a given set of gases and operational environments is not yet mature. This includes a lack of a clear understanding of sensing mechanisms and correlation of sensing properties to device processing. Significant further work is necessary towards the realization of repeatable, controlled sensor systems composed of oxide based nanostructures.

\section{Acknowledgements}

We gratefully acknowledge the contributions of those who made this paper and its contents possible: Dr. L. Matus, Dr. M. Zeller, Dr. Carl Chang, Gustave Fralick, and Dr. Michael Lienhard, and C. Swanson of NASA GRC, D. Androjna, M. Artale, P. Lampard of Sierra Lobo/NASA GRC

\section{References}

1. G.W. Hunter, J.C. Xu, and D.B. Makel, "Case Studies in Chemical Sensor Development", Chapter 9, in BioNanoFluidic MEMS, P. Hesketh Ed. Springer Press, New York, NY, (2007).

2. G.W. Hunter, J.C. Xu, C.C. Liu, and D.B. Makel, in The MEMES Handbook Second Edition: Design and Fabrication, M. Gad-El-Hak, Editor, Chapter 11, CRC Press, Baton Rouge (2006).

3. G.W. Hunter, J.C. Xu, L.J. Evans, R.L. Vander Wal, G.M. Berger, M.J. Kulis, and C.C. Liu, "Chemical Sensors Based on Metal Oxide Nanostructures", ECS Transactions, 3 (9) 199-209 (2006).

4. C.C. Liu, P.J. Hesketh, and G.W. Hunter, The Electrochemical Society Interface Magazine, 13 22-29 (2004).

5. P.A. Smith, C.D. Nordquist, T.N. Jackson, T.S. Mayer, B.R. Martin, J. Mbindyo, T.E. Mallouk, "Electric-Field Assisted Assembly and Alignment of Metallic Nanowires" Appl. Phys. Lett. 77, 1399-1401 (2000).

6. A. Tselev, K. Hatton, M.S Fuhrer, M. Paranjape, and P. Barbara, "A photolithographic process for fabrication of devices with isolated single-walled carbon nanotubes", Nanotechnology 15, 1475-1478 (2004).

7. S. Jin, D. Whang, M. C. McAlpine, R. S. Friedman, Y. Wu, and C. M. Lieber, "Scalable Interconnection and Integration of Nanowire Devices without Registration", Nanoletters, 4, 915-919 (2004).

8. A. Subramanian, B. Vikramaditya, B. J. Nelson, D. Bell, and D. Lixin, "Dielectrophoretic micro/nanoassembly with microtweezers and nanoelectrodes", Advanced Robotics, ICAR '05. Proceedings, 12th International Conference, 208- 215 (2005).

9. Z. Fan and J.G. Lu, "Zinc Oxide Nanostructures: Synthesis and Properties", J. Nanosci. Nanotechnol. 5 (10), 1561-1573 (2005).

10. Alumina substrate, $625 \mu \mathrm{m}$ in thickness (CoorsTec).

11. S. E. Mohney, Y. Wang, M.A. Cabassi, K.K. Lew, S. Dey, J.M. Redwing, T.S. Mayer, "Measuring the Specific Contact Resistance Of Contacts to Semiconductor Nanowires", Solid State Electronics, 49, 227-232 (2005). 